\title{
El biomodelo porcino en la investigación médica traslacional: del biomodelo al humano en trasplante pulmonar
}

Liliana Fernández-Trujillo, 2,3,3, Mauricio Velázquez ${ }^{3,4,5}$, Luz Fernanda Sua ${ }^{2,3,4,6}$, Indira Cujiño $3,4,7$, Martha Giraldo 7 , Diego Medina ${ }^{8}$, Mauricio Burbano ${ }^{6}$, Germán Torres 6 , Carlos Muñoz-Zuluaga ${ }^{2,3,4}$, Leidys Gutiérrez-Martínez $2,3,4$

${ }^{1}$ Departamento de Medicina Interna, Servicio de Neumología, Fundación Valle del Lili, Cali, Colombia ${ }^{2}$ Centro de investigaciones Clínicas, Fundación Valle del Lili, Cali, Colombia

${ }^{3}$ Línea de Investigación Biomédica en Tórax, Fundación Valle del Lili, Cali, Colombia

${ }^{4}$ Facultad de Ciencias de la Salud, Universidad Icesi, Cali, Colombia

${ }^{5}$ Unidad de Cirugía de Tórax, Fundación Valle del Lili, Cali, Colombia

${ }^{6}$ Departamento de Patología y Medicina de Laboratorio, Fundación Valle del Lili, Cali, Colombia

${ }^{7}$ Servicio de Anestesiología, Fundación Valle del Lili, Cali, Colombia

${ }^{8}$ Unidad de Cirugía Cardiovascular, Fundación Valle del Lili, Cali, Colombia

${ }^{9}$ Servicio de Hemato-Oncología Pediátrica, Fundación Valle del Lili, Cali, Colombia

Introducción. La anatomía humana y porcina son comparables. En consecuencia, el biomodelo porcino tiene el potencial de ser implementado para entrenar al profesional quirúrgico en áreas como el trasplante de órganos sólidos.

Objetivo. Describir los procedimientos y hallazgos obtenidos mediante experimentos de medicina respiratoria traslacional con biomodelos porcinos realizados en un laboratorio de experimentación animal, y hacer una revisión comparativa entre el pulmón humano y el porcino.

Materiales y métodos. El experimento se llevó a cabo en nueve cerdos de raza híbrida en un laboratorio de cirugía experimental. Se estudiaron la anatomía y la histología de las vías respiratorias mediante fibrobroncoscopia, biopsia bronquial y lavado broncoalveolar. El lavado broncoalveolar se estudió con citología en base líquida y se evaluó con las coloraciones de Papanicolau y hematoxilina y eosina. Se utilizaron técnicas de patología molecular, como inmunohistoquímica, citometría de flujo y microscopía electrónica. Los cerdos se sometieron a neumonectomía izquierda con posterior implante del injerto en otro cerdo experimental.

Resultados. Los estudios histopatológicos y moleculares evidenciaron un predominio de macrófagos alveolares (98 \%) y linfocitos T (2\%) en el lavado broncoalveolar porcino. En los estudios del parénquima pulmonar porcino se encontró tejido linfoide hiperplásico asociado a las paredes bronquiales. La microscopía electrónica evidenció linfocitos T dentro del epitelio y el diámetro de las cilias porcinas fue similar al de las humanas. Conclusiones. El biomodelo porcino es viable en la investigación traslacional para el entendimiento de la anatomía del sistema respiratorio y el entrenamiento en trasplante pulmonar. La implementación de este modelo experimental podría fortalecer los grupos que planean implementar un programa institucional de trasplante pulmonar en humanos.

Palabras clave: trasplante de pulmón; modelos animales; histología comparada; citometría de flujo, médula ósea.

The porcine biomodel in translational medical research: From biomodel to human lung transplantation

Introduction: Human and porcine anatomy are comparable. In consequence, the porcine biomodel has the potential to be implemented in the training of surgical professionals in areas such as solid organ transplantation.

Objectives: We described the procedures and findings obtained in the experiments of translational respiratory medicine with the porcine biomodel, within an experimentation animal laboratory, and we present a comparative review between human and porcine lung. Materials and methods: The experiment was done in nine pigs of hybrid race within a laboratory of experimental surgery. The anatomy and histology of the respiratory tract were studied with fibrobronchoscopy, bronchial biopsy and bronchoalveolar lavage. The bronchoalveolar lavage was studied with liquid-based cytology and assessed with Papanicolau and hematoxylin-eosin staining. Molecular pathology techniques such as immunohistochemistry, flow cytometry, and electronic microscopy were implemented. The pigs were subjected to left pneumonectomy with posterior implantation of the graft into another experimental pig. 
Results: Histopathologic and molecular studies evidenced predominance of alveolar macrophages (98\%) and T-lymphocytes $(2 \%)$ in the porcine bronchoalveolar lavage. Studies on the porcine lung parenchyma revealed hyperplasic lymphoid tissue associated with the bronchial walls. Electronic microscopy evidenced the presence of T-lymphocytes within the epithelium and the cilia diameter was similar to the human.

Conclusions: The porcine biomodel is a viable tool in translational research applied to the understanding of the respiratory system anatomy and the training in lung transplantation. The implementation of this experimental model has the potential to strength the groups who plan to implement an institutional program of lung transplantation in humans.

Keywords: Lung transplantation; models, animals; histology, comparative; flow cytometry; bone marrow.

Los modelos animales son fundamentales para el entendimiento de la enfermedad y en la evaluación preclínica de la seguridad y la eficacia de nuevas alternativas terapéuticas. La similitud anatómica y fisiológica entre el cerdo y el humano ha permitido emplear esta especie como un valioso modelo para la investigación biomédica traslacional (1).

En el campo de la medicina respiratoria, el biomodelo porcino ha permitido estudiar enfermedades como la fibrosis quística, la enfermedad pulmonar obstructiva crónica y las infecciones respiratorias (2-4). Asimismo, en estudios previos se han documentado exitosamente mecanismos inmunológicos humanos en el biomodelo porcino, lo cual aumenta su potencial como posible modelo para la investigación biomédica en trasplantes (5).

Por otro lado, el trasplante pulmonar es la única opción viable para los individuos con enfermedad pulmonar avanzada en estado terminal. En Colombia, la escasa disponibilidad de donantes óptimos y la poca frecuencia de trasplantes en humanos para el perfeccionamiento de la técnica en un equipo multidisciplinario, que involucra cirujanos, neumólogos y patólogos, limitan el uso de este procedimiento. Previamente, se han empleado biomodelos porcinos en diversos centros hospitalarios para adquirir experiencia mediante protocolos experimentales de trasplante pulmonar $(6,7)$.

En el Laboratorio de Cirugía Experimental y Cirugía Avanzada, CICAT (Cali, Colombia), desde el 2012 se empezaron a utilizar cerdos como biomodelos experimentales de trasplante pulmonar. En el contexto nacional, la experiencia descrita es limitada. En consecuencia, en este artículo se describen los procedimientos y hallazgos obtenidos en los experimentos de trasplante pulmonar con biomodelos porcinos llevados a cabo en un laboratorio de experimentación animal. Además, se revisa la literatura científica comparativa entre el pulmón porcino y el humano.

\section{Materiales y métodos}

El experimento se llevó a cabo en nueve cerdos machos y hembras jóvenes de la raza híbrida Large White y Landrace, que pesaban entre 30 y $40 \mathrm{~kg}$, con un ayuno de más de 12 horas, los cuales habían sido evaluados previamente por el médico veterinario.

Se sometieron a neumonectomía izquierda e implante secuencial del injerto en otro sujeto de experimentación, según el protocolo de trasplante pulmonar porcino. Se hicieron registros del comportamiento animal, la anestesia y la cirugía, la técnica, el instrumental, los tiempos, los problemas y eventos no esperados, y la variabilidad de las mediciones. Se describieron los detalles del procedimiento anestésico, la cirugía y la evaluación endoscópica con broncoscopia, así como los hallazgos histológicos, moleculares y hematológicos obtenidos en muestras de tejido pulmonar, del lavado broncoalveolar y de la médula ósea. 
Los animales ingresaron al bioterio dos o tres días antes del procedimiento, permanecieron en observación y fueron evaluados constantemente por el personal veterinario. Durante este lapso de tiempo se vigiló la aparición de alteraciones del comportamiento o del estado general que pudieran contraindicar la continuación del experimento.

\section{Anestesia}

El protocolo de sedación del cerdo se inició con $10 \mathrm{mg} / \mathrm{kg}$ de ketamina por vía intramuscular, repitiendo la dosis cada 20 minutos según la necesidad, mientras el animal se trasladaba desde el bioterio y se instalaba en el quirófano.

Posteriormente, se canalizó la vena auricular media de la oreja, se instaló monitorización básica (electrocardiograma, oximetría de pulso y tensión arterial) más monitorización invasiva continua de la tensión arterial, y se ubicó el biomodelo sobre la mesa en decúbito prono, dado que el manejo de la vía aérea y la intubación son más fáciles en esta posición.

Se utilizó sedación sin relajación y con preoxigenación adecuada, se procedió a abrir la mandíbula mediante dos cordeles de sostenimiento, uno atado al maxilar superior y otro en la mandíbula. Después, se introdujo un laringoscopio con hoja recta y se avanzó un tubo de una luz del mayor diámetro posible (entre 7 y $7,5 \mathrm{~mm}$ ).

Tras verificar la posición endotraqueal del tubo con capnografía, se calcularon los parámetros del respirador con una frecuencia respiratoria de 12 a 14 respiraciones por minuto, volumen corriente de $6 \mathrm{ml} / \mathrm{kg}$ y presión positiva al final de la espiración (Positive End Expiratory Pressure, PEEP) de $5 \mathrm{~cm} \mathrm{H}_{2} \mathrm{O}$.

El mantenimiento anestésico se llevó a cabo con infusión continua de propofol (0,1-0,2 mg/kg/minuto), fentanilo (1-2 $\mu \mathrm{g} / \mathrm{hora})$ y Pavulon ${ }^{\mathrm{TM}}(0,1 \mathrm{mg} /$ hora según necesidad).

Posteriormente, se canalizó la arteria auricular mediante disección, se midieron los gases arteriales y se corrigieron los parámetros respiratorios según el caso y las condiciones clínicas del animal.

Se administró profilaxis antibiótica según el protocolo y se recolectó sangre para autotransfusión. Los líquidos de mantenimiento durante el procedimiento quirúrgico se administraron a razón de $3 \mathrm{ml} / \mathrm{kg} / \mathrm{hora}$, las pérdidas por evaporación y respiración a razón de $5 \mathrm{ml} / \mathrm{kg}$ por hora y el sangrado intraoperatorio se reemplazó en una relación de 3:1 con cristaloides y de 2:1 con coloides.

\section{Broncoscopia}

Después de garantizar una adecuada profundidad anestésica durante el experimento, se hizo una broncoscopia flexible con un equipo Olympus ${ }^{\mathrm{TM}}$ de $4,5 \mathrm{~mm}$ de diámetro a través del tubo orotraqueal. Se evaluó la segmentación pulmonar y se tomaron muestras de tejido y lavado broncoalveolar con las mismas técnicas utilizadas en humanos, sin que se presentaran complicaciones (figura 1).

Las biopsias bronquiales y transbronquiales se obtuvieron mediante diferentes tipos de pinzas y, para el lavado bronquial o broncoalveolar, la técnica incluyó la aplicación de alícuotas de 10 a $20 \mathrm{ml}$ de solución salina normal hasta $200 \mathrm{ml}$, que se aspiraron mediante una trampa de material (8) 


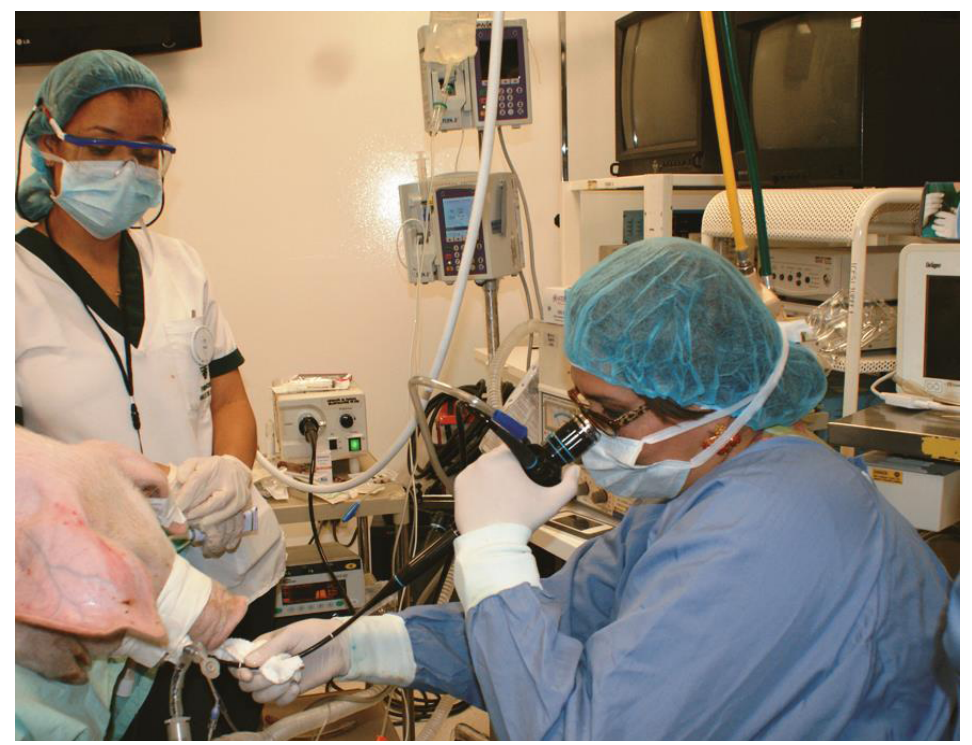

Figura 1. Estudio de la vía aérea porcina con fibrobroncoscopia flexible y toma de muestras de lavado broncoalveolar

habiendo pasado el broncoscopio a través del tubo orotraqueal sin aspirar secreciones antes de acuñarlo distalmente, para evitar la contaminación de la muestra con secreciones altas.

El lavado bronquial permite remover células no adheridas y fluido de la superficie bronquial, mientras que el lavado broncoalveolar remueve macrófagos, células $B$ y $T$, inmunoglobulinas y microorganismos presentes en el árbol traqueobronquial bajo (8).

\section{Extracción de médula ósea}

Antes de iniciar el procedimiento quirúrgico, con el cerdo en sedación profunda, se tomó una muestra de la médula ósea mediante aspiración de la cresta iliaca izquierda para el análisis de citometría de flujo y los estudios comparativos con la médula ósea humana, según el estándar utilizado en humanos.

\section{Técnica quirúrgica}

Explante pulmonar. El protocolo de cirugía se inició con la colocación del cerdo en decúbito supino. Después de una cuidadosa limpieza de la piel, se procedió con la incisión de una esternotomía media. El primer procedimiento dentro de la cavidad torácica fue la apertura de las pleuras para la inspección de los pulmones, seguida de la apertura del pericardio y la inspección y disección de la arteria pulmonar. Posteriormente, se disecó el ligamento pulmonar izquierdo, paso que puede ser difícil porque el abordaje es profundo en el espacio costodiafragmático.

Una vez el pulmón estuvo libre, se inició la disección del hilio empezando por las dos venas pulmonares, una craneal y otra caudal, las cuales se encuentran en el plano anterior más superficial del mediastino y tienen un curso extrapericárdico que favorece la disección en bloque. Estos vasos son muy frágiles en el biomodelo porcino y se lesionan fácilmente. Se continuó con la disección de la arteria pulmonar y la vena hemiácigos izquierda -que es prominente en los modelos porcinos- se disecó y se ligó. A continuación, se procedió a disecar el bronquio hasta donde fuera posible, dadas las características anatómicas del biomodelo. 
Enseguida, se procedió a la infusión de la dosis de heparina, calculada según el peso del animal (3 mg/kg), y a fabricar una jareta con proleno 5-0 en la cara anterior de la arteria pulmonar, y se introdujo una cánula venosa № 12 .

Con el fin de practicar la neumonectomía y el explante del órgano, se procedió a pinzar la aorta torácica y se inició la infusión del líquido de preservación. La solución de preservación utilizada fue perfadex ${ }^{T M}(60 \mathrm{ml} / \mathrm{kg}$ de peso del biomodelo), con descompresión del sistema mediante una incisión en la vena cava inferior y la orejuela cardiaca izquierda. Las bolsas de la solución se ubicaron a una altura suficiente ( $\pm 50 \mathrm{a} 60 \mathrm{~cm}$ por encima del tórax) para mantener una presión de perfusión de 15 a $20 \mathrm{~mm} \mathrm{Hg}$. Se anotó la hora en que se colocaron las pinzas y se controló que hubiera buena perfusión.

En este punto de la extracción comenzó el tiempo de isquemia fría, durante el cual es importante mantener la superficie pulmonar a la menor temperatura posible, por lo que a la baja temperatura de la solución de perfadex ${ }^{\mathrm{TM}}$, se añadió la instilación continua de suero frío en ambos hemitórax $\left(\mathrm{a}^{\circ}{ }^{\circ} \mathrm{C}\right)$. Hasta ese momento se mantuvieron los pulmones ventilados con una fracción de $\mathrm{O}_{2}$ inspirado $\left(\mathrm{FIO}_{2}\right)$ de 0,4 o lo suficiente para mantener la saturación entre $95 \mathrm{y}$ $100 \%$ y la PEEP de 3 a $5 \mathrm{~cm}$ de $\mathrm{H}_{2} \mathrm{O}$.

Se fabricaron los muñones de la arteria pulmonar y de las venas pulmonares, dejando una orejuela de aurícula izquierda para facilitar la anastomosis, así como el muñón bronquial. Se extrajo el pulmón y, ya fuera del campo, se comprobó el correcto estado de las estructuras. Se introdujo una cánula en las venas pulmonares para hacer la perfusión pulmonar retrógrada con solución de perfadex $^{\text {TM }}$ hasta que el líquido que saliera por la arteria pulmonar fuera totalmente cristalino. Se introdujo el pulmón en la bolsa de plástico estándar con el líquido de perfusión restante y se cerró herméticamente. A continuación, se introdujo en una segunda bolsa con suero frío y luego en una tercera, según la recomendación del protocolo convencional del manejo del órgano donado en trasplante pulmonar.

Implante pulmonar. En la cirugía de implante pulmonar en otro biomodelo porcino se siguieron los pasos de la anestesia del modelo de una forma similar al de la extracción pulmonar y, luego, se inició la monitorización invasiva, para lo cual se colocó un catéter en la arteria pulmonar mediante disección por la vía yugular externa, el cual se avanzó hasta la salida de la arteria pulmonar. Se introdujo, también, una sonda vesical Foley № 12 para la medición horaria de la diuresis. Al inicio, se extrajeron $200 \mathrm{ml}$ de sangre total del biomodelo receptor en la bolsa de preservación para la autotransfusión, con el fin de utilizarlos en la fase de reperfusión.

Durante el proceso del implante, se aisló el pulmón izquierdo por medio de la ventilación del pulmón derecho, avanzando el tubo orotraqueal hasta el bronquio fuente derecho. Se elevó la frecuencia respiratoria a 18 respiraciones por minuto y se disminuyeron los volúmenes pulmonares a 4-5 $\mathrm{ml} / \mathrm{kg}$, manteniendo las presiones pico en la vía aérea por debajo de 25 $\mathrm{cm}$ de $\mathrm{H}_{2} \mathrm{O}$. Se midieron los gases arteriales a los 10 minutos de iniciada la ventilación unipulmonar y se hicieron los correctivos correspondientes.

El protocolo de la cirugía se inició colocando al cerdo en decúbito lateral derecho. Se hizo una cuidadosa limpieza de la piel, se procedió con la incisión de toracotomía postero-lateral izquierda desde el primer pezón hasta $2 \mathrm{~cm}$ por debajo de la punta de la escápula y luego postero-cranealmente alrededor de la escápula, $5 \mathrm{~cm}$ aproximadamente. La incisión en la piel fue de $17 \pm 2 \mathrm{~cm}$ en el séptimo espacio intercostal, disecando por planos hasta llegar a la cavidad pleural. 
El primer procedimiento dentro de la cavidad fue la disección del ligamento pulmonar. Se continuó con la disección del hilio, empezando por las venas pulmonares y siguiendo con la arteria pulmonar, y luego con la disección del bronquio para completar la neumonectomía y extraer el pulmón izquierdo. Después de esto, se extrajo el pulmón izquierdo de la nevera y las bolsas de preservación y se introdujo en la cavidad pleural. Se hicieron las anastomosis de aurícula izquierda y la arteria pulmonar con prolene 5-0 y, la del bronquio, con prolene 4-0. Se administró un gramo de metilprednisolona para inducir la inmunosupresión. Se acomodó el biomodelo receptor en la posición de Trendelemburg y se retiró la pinza de la aurícula para perfundir nuevamente el pulmón en sentido retrógrado, y dejar salir el aire y los restos del líquido de reperfusión a través del último punto de la sutura arterial antes de anudarla. Posteriormente, se abrió muy lentamente la arteria para evitar el aumento brusco de la presión en el lecho vascular del pulmón donante, que estaba frío y bajo los efectos de la isquemia.

Una vez se completó el proceso de implante y reperfusión del pulmón, se retiró nuevamente el tubo orotraqueal hasta la tráquea y se reanudó la insuflación de ambos pulmones, primero manualmente y, después, con el respirador, monitorizando las presiones en la vía aérea y en los pulmones, así como los parámetros gasométricos. Se vigiló constantemente la volemia y se repuso con cristaloides y la sangre para autotransfusión almacenada previamente. La monitorización hemodinámica mostró la necesidad de utilizar fenilefrina en bolos de $0,01 \mathrm{mg}$ o en infusión por microgoteo cuando se presentaba hipotensión sostenida.

Se llevaron a cabo la hemostasia, el cuidado, la revisión y el lavado con suero de la cavidad pleural. Seguidamente, se colocó un tubo de tórax $\mathrm{N}^{\circ}$ 28 , el cual se fijó con seda 0 y se cerró por planos, según lo habitual en una cirugía de tórax.

Se hizo un control broncoscópico al final de la intervención para comprobar el estado de la sutura bronquial y limpiar de secreciones el árbol bronquial. Se suministró soporte de oxígeno para garantizar saturaciones superiores al 90 $\%$; se manejó el dolor y se controlaron el cuadro hemático, los gases arteriales cada seis horas, el volumen urinario y la producción por el tubo de tórax.

El protocolo de experimentación estipulaba el sacrificio 24 horas después del procedimiento y el explante del pulmón trasplantado para su estudio microscópico. Los biomodelos se conservaron por un periodo máximo de seis horas (9).

\section{Estudios de patología molecular}

El lavado broncoalveolar se estudió por citología en base líquida ( $B D$ CytoRich ${ }^{\mathrm{TM}}$ ), según el protocolo del laboratorio institucional, y se evaluó con coloraciones de Papanicolau, y de hematoxilina y eosina. La medula ósea se fijó en TransFix ${ }^{\mathrm{TM}}$ y se transportó inmediatamente al laboratorio de citometría de flujo.

Se observó la distribución celular medular según el tamaño y la granularidad (SS INT/FS INT) y se evaluó la expresión del anticuerpo CD34 en las células madre de medula ósea. Se evaluó el parénquima pulmonar con la coloración de hematoxilina y eosina.

Por último, el epitelio bronquial se estudió mediante microscopía electrónica de barrido. 


\section{Consideraciones éticas}

Este estudio se rigió por la Ley 84 de 1989, es decir, por el Estatuto Nacional de Protección de Animales y por la Declaración Universal de los Derechos del Animal. El protocolo de investigación fue evaluado y aprobado por el Comité de Ética para la experimentación animal de la Universidad Icesi.

\section{Resultados}

\section{Lavado broncoalveolar}

Se encontró predominio de macrófagos alveolares (98 \%) y linfocitos (2\%). No se encontraron neutrófilos, cristales ni microorganismos (figura 2).

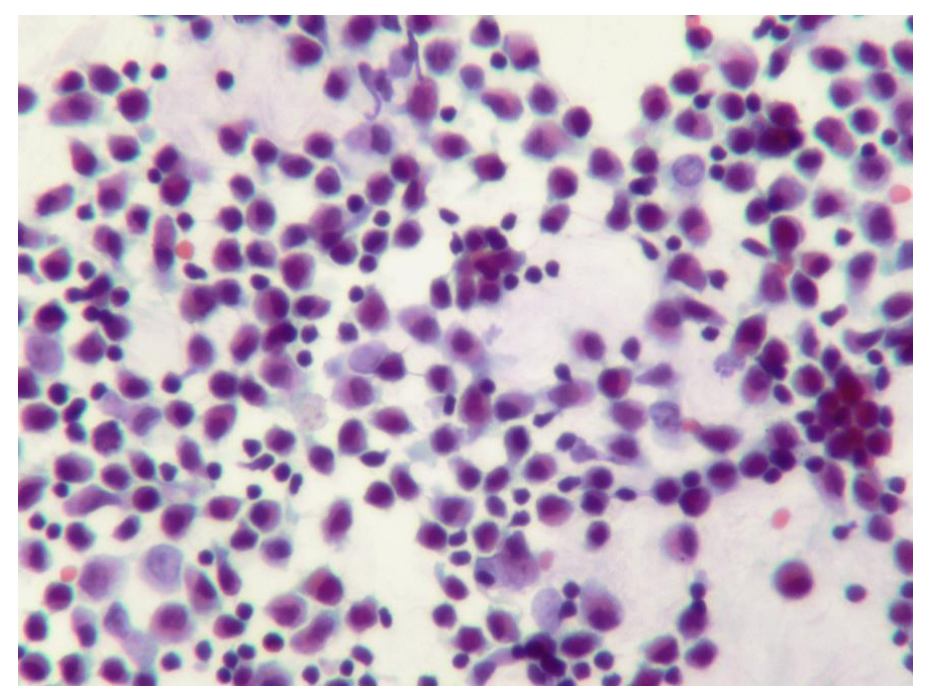

Figura 2. Lavado broncoalveolar con predominio (98\%) de macrófagos alveolares. Papanicolau, 40X

\section{Médula ósea}

En la médula ósea y en los estudios de citometría de flujo se evidenció viabilidad adecuada y una posición similar a la humana en la gráfica de puntos (figura 3).

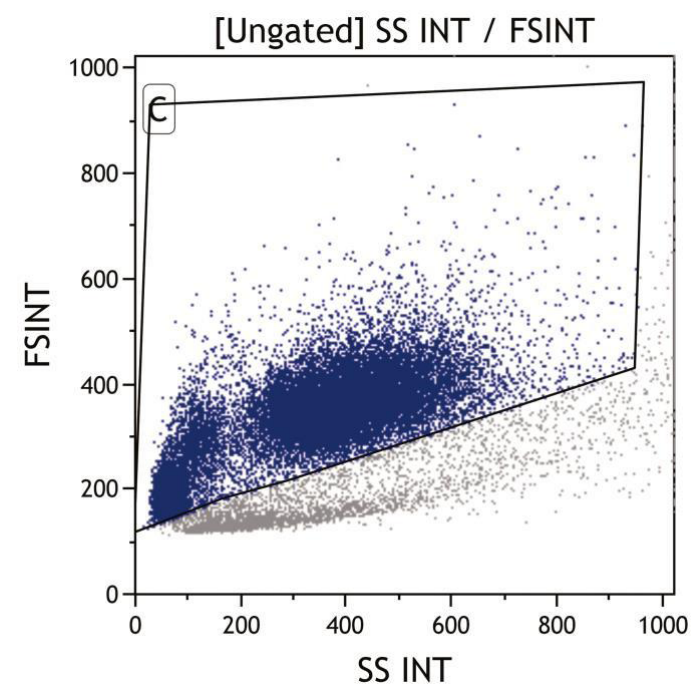

Figura 3. Distribución según tamaño y granularidad de la medula ósea porcina. Según la citometría de flujo 


\section{Parénquima pulmonar}

En el cerdo se encontró un sistema de conducción, transición e intercambio, así como bronquios, bronquiolos, alvéolos, trama vascular y distribución de neumocitos de tipo 1 y de tipo 2, similares a los de los humanos. Además, se observó tejido linfoide hiperplásico asociado a las paredes bronquiales (Bronchus-Associated Lymphoid Tissue, BALT), el cual solo se observa en enfermedades respiratorias como la bronquiolitis folicular en los humanos (figura 4).

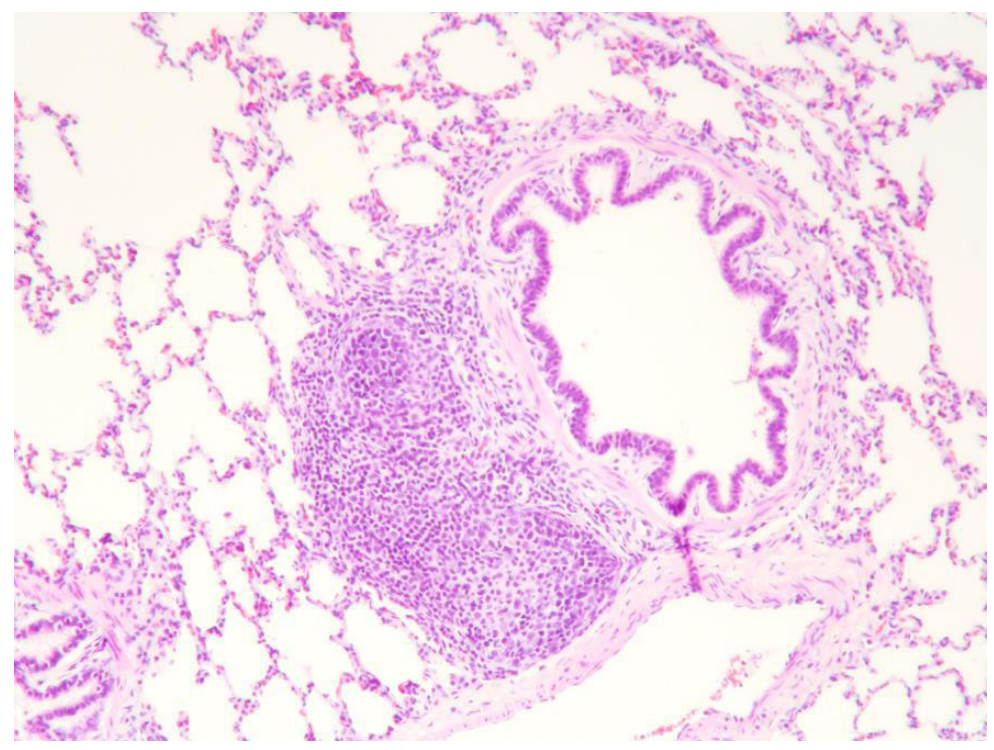

Figura 4. Presencia de BALT en tejido pulmonar porcino, normal en el cerdo pero no en el humano. Coloración hematoxilina-eosina. 10x

\section{Inmunohistoquímica}

En cuanto a la expresión de anticuerpos, se comprobó una expresión similar a la humana de citoqueratinas en el epitelio respiratorio, entre ellas, la CKAE1/AE3. Asimismo, hubo expresión de CD3 o CD34, según el cuadro, en los linfocitos de la línea $T$ de los cerdos, antígeno que también se expresa en el humano (cuadro 1).

Cuadro 1. Características anatomopatológicas del biomodelo porcino de la especie híbrida Large White y Landrace y del humano

\begin{tabular}{|c|c|c|}
\hline $\begin{array}{l}\text { Características } \\
\text { anatomopatológicas }\end{array}$ & Biomodelo porcino & Humano \\
\hline $\begin{array}{l}\text { Sistema de conducción, transición } \\
\text { e intercambio }\end{array}$ & Similar & Similar \\
\hline $\begin{array}{l}\text { Bronquio, bronquiolos, alvéolos y } \\
\text { trama vascular }\end{array}$ & Similar & Similar \\
\hline $\begin{array}{l}\text { Distribución de neumocitos de tipo } \\
1 \text { y de tipo } 2\end{array}$ & Similar & Similar \\
\hline $\begin{array}{l}\text { Tejido linfoide asociado a los } \\
\text { bronquios (Bronchus-Associated } \\
\text { Lymphoid Tissue, BALT) }\end{array}$ & Hiperplásico & No hiperplásico \\
\hline Leucocitos & $11.000-22.000$ células $/ \mu l$ & $5.000-10.000$ células $/ \mu l$ \\
\hline $\begin{array}{l}\text { Cuantificación y viabilidad de las } \\
\text { células madre por citometría de flujo }\end{array}$ & $\begin{array}{l}\text { Expresión, viabilidad y } \\
\text { posición similar de CD34 }\end{array}$ & $\begin{array}{l}\text { Expresión, viabilidad y } \\
\text { posición similar de CD34 }\end{array}$ \\
\hline
\end{tabular}




\section{Microscopía electrónica de barrido}

En el estudio con microscopía electrónica de barrido, se encontraron linfocitos $T$ dentro del epitelio respiratorio y, además, cilias con un diámetro entre 12 y $14 \mu \mathrm{m}$, como en los humanos (figura 5).

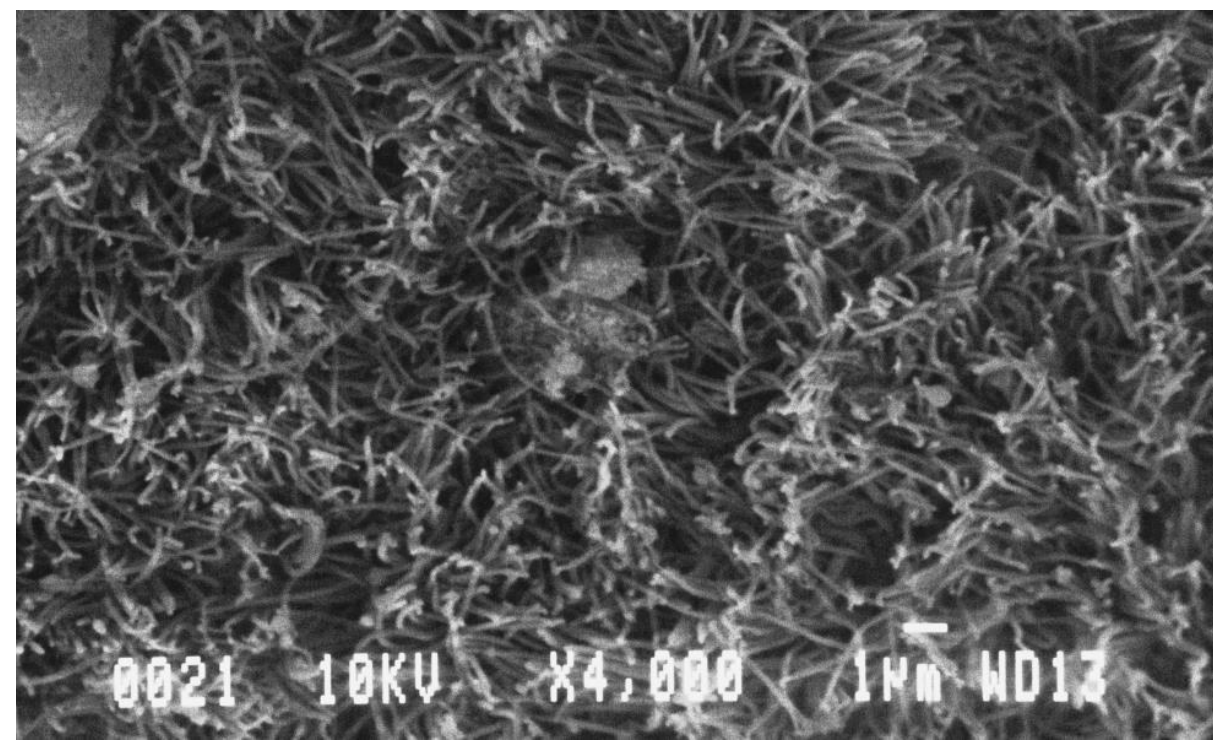

Figura 5. Microscopía electrónica de barrido donde se observan cilias con un diámetro de 12 a 14 $\mu \mathrm{m} .4 .000 \mathrm{X}$

\section{Discusión}

En investigación biomédica traslacional, el biomodelo porcino es valioso para el estudio de enfermedades humanas de interés gracias a su similitud con el ser humano en cuanto a la anatomía, histología y algunos mecanismos fisiopatológicos $(1,10)$.

Recientemente, la limitación para hacer comparaciones con modelos de roedores ha sido superada gracias al cerdo y los notables avances en el estudio de su genoma, así como en la generación de modelos porcinos en los cuales se pueden recrear muchas de las enfermedades genéticas del ser humano para su estudio (1). Estos logros han aumentado considerablemente la importancia del biomodelo porcino en la investigación biomédica traslacional. Como resultado de lo anterior, el cerdo se ha implementado como biomodelo para la fibrosis quística y el enfisema pulmonar, y ha sido útil en el estudio y la validación de nuevos tratamientos $(2,3)$.

En el área del trasplante pulmonar, el cerdo ha sido el animal grande más comúnmente usado en investigación $(11,12)$. Este biomodelo tiene el potencial de ser implementado en el aprendizaje y el reforzamiento de la técnica quirúrgica, el estudio del procedimiento anestésico, los procedimientos invasivos y el sostenimiento posoperatorio $(7,9,13)$. Además, ofrece la posibilidad de integrar desde el contexto experimental el trabajo multidisciplinario que se exige en los trasplantes durante el entrenamiento previo al ejercicio médico real.

El trasplante pulmonar es una opción terapéutica definitiva para los pacientes con enfermedades pulmonares irreversibles y terminales (14). Además, en el contexto nacional, los estudios sobre experimentación animal con enfoque práctico son limitados. En este sentido, este estudio brinda 
evidencia sobre la viabilidad de ejecutar el trasplante pulmonar en el biomodelo porcino y la posibilidad de fortalecer esta práctica a nivel institucional, así como la integración del equipo que interviene en dicho procedimiento.

Son múltiples las similitudes anatómicas e histológicas entre el sistema respiratorio porcino y el del ser humano, las cuales ofrecen importantes ventajas al momento de considerar los modelos animales de experimentación en los estudios de medicina respiratoria traslacional.

A continuación, se hace una revisión comparativa en la que se describen las similitudes anatómicas e histológicas entre el sistema respiratorio porcino y el humano, las cuales permiten considerar el biomodelo porcino como ventajoso con respecto a otros modelos animales de experimentación.

\section{Anatomía del pulmón porcino}

A diferencia de los bronquiolos respiratorios humanos que a menudo están conectados a dos o tres conductos alveolares primordiales en el nacimiento, los bronquiolos respiratorios porcinos neonatales están típicamente conectados con los conductos alveolares (15).

Los alvéolos porcinos se multiplican rápidamente en las primeras dos a cuatro semanas de vida, mientras que el desarrollo es similar durante los tres primeros años de la vida humana $(15,16)$. El tamaño de los acinos porcinos perinatales $(\sim 5 \mathrm{~mm})$ es más pequeño que el de los acinos del infante humano $(\sim 11 \mathrm{~mm})(7)$.

La estructura y la distribución de las vías respiratorias porcinas varían morfológicamente según la edad y la raza de cerdo, pero son muy similares a las del pulmón humano. La tráquea porcina es notablemente más larga y más cartilaginosa que en los seres humanos; esta contiene 32 a 45 anillos y se divide en el bronquio fuente derecho y en el izquierdo a nivel de la quinta vértebra dorsal.

En el cerdo las vías respiratorias contienen más cartílago en su estructura, pero tienen un número similar de generaciones bronquiales: se han identificado 23 en los seres humanos y en los cerdos $(15,17,18)$. La disminución general del diámetro y la longitud de las bifurcaciones del árbol bronquial humano también se observan en las vías respiratorias porcinas $(18,19)$. Sin embargo, en contraste con el árbol de las vías respiratorias humanas, que tiene un patrón de ramificación bípoda, el porcino tiene un patrón de ramificación monópoda en el cual cada bronquio 'madre' da lugar a ramas laterales más pequeñas (bronquios) que se ramifican en ángulos obtusos (20).

Los pulmones de los cerdos y de los humanos son lobulados, con el lóbulo pulmonar bien definido y demarcado por tabiques interlobulillares. En el pulmón humano, el componente de colágeno de estos tabiques interlobulares es incompleto, y se han descrito poros interalveolares, o de Kohn, y canales de comunicación en las paredes alveolares $(21,22)$. Estos factores pueden conducir a la ventilación colateral entre los lóbulos, especialmente en los pulmones enfisematosos. Por el contrario, el componente de colágeno de los tabiques interlobulares porcinos es más completo y la ventilación colateral es sustancialmente menos probable (23).

La anatomía bronquial y lobar de los cerdos es similar a la de los humanos. El pulmón izquierdo porcino es similar al pulmón izquierdo humano, ya que consta de un lóbulo craneal y un lóbulo caudal. El bronquio del lóbulo craneal izquierdo se divide en bronquios segmentarios craneal 
y caudal. Esto es similar a la división de los bronquios del lóbulo superior izquierdo, para el lóbulo superior izquierdo y la língula, en los seres humanos. El bronquio izquierdo caudal se divide en cuatro bronquios segmentarios ventrales y cuatro dorsales, que ventilan el lóbulo caudal izquierdo.

En contraste con el pulmón derecho humano que tiene tres lóbulos, el pulmón porcino derecho se divide en cuatro lóbulos (craneal, medio, accesorio y caudal). El lóbulo craneal derecho es servido por un bronquio craneal (traqueal), el cual surge de la pared derecha de la tráquea, proximal a la bifurcación de la tráquea de manera independiente. El bronquio del lóbulo craneal se subdivide en los bronquios segmentarios craneal y caudal (24). Esta variante anatómica hace que el trasplante pulmonar derecho sea técnicamente difícil, por lo cual en nuestros biomodelos se seleccionó el pulmón izquierdo para el procedimiento.

Los bronquios traqueales son alteraciones que también se encuentran en los seres humanos y, por lo general, surgen entre los 2 y los $6 \mathrm{~cm}$ de la carina (25). El bronquio medio derecho porcino, que ventila el lóbulo medio derecho, surge inmediatamente caudal a la bifurcación de la tráquea en el lado ventrolateral. El bronquio derecho accesorio, que ventila el pequeño lóbulo accesorio, surge justo caudal al bronquio del lóbulo medio y tiene un recorrido ventromedial (26). El lóbulo accesorio está situado adyacente a la base del corazón y el diafragma, y rodea la porción intratorácica terminal de la vena cava caudal (26). El lóbulo caudal derecho se ventila por el bronquio caudal derecho, que se divide en cuatro bronquios segmentarios dorsales y cuatro ventrales (27).

La anatomía bronquial del cerdo se ha clasificado en sistemas bronquiolares dorsal, lateral, ventral y medial, cada uno nombrado según el orden en que los bronquiolos provienen del bronquio principal en cada lado (24). Aquí se propone un sistema similar al que se ha utilizado en ambos campos, el clínico y el de investigación, para los perros, caballos, gatos y seres humanos $(28,29)$. Cada bronquio lobar se denomina según el lado del pulmón que se ventila y según el orden en el que se encuentra en la broncoscopia (30). Por consiguiente, el bronquio del lóbulo craneal derecho se llama RB1; el lobar medio, RB2; el accesorio del lóbulo derecho, RB3, y el caudal, RB4. En el pulmón izquierdo, el bronquio craneal y el caudal se denominan LB1 y LB2, respectivamente. Las ramas de los bronquios lobares se nombran según su orientación predominante así: dorsal [D], ventral [V], craneal [Cr], caudal [Cd] con el fin de proximal a distal en letras minúsculas $[a, b$, etc.] que representan bronquios subsegmentarios, por ejemplo, RB1, RB1Cr1, RB1Cr1a, etc. (30).

La arteria pulmonar se divide en rama derecha e izquierda, tiene un curso dorso-lateral acompañando el bronquio correspondiente, y se divide en concordancia con el árbol bronquial; las venas pulmonares se localizan ventromediales a las estructuras bronquiales. El sistema vascular sistémico tiene diferencias en el modelo porcino: la vena hemiácigos izquierda cursa al lado de la aorta descendente en el mediastino posterior y, después, cruza el hilio pulmonar izquierdo para drenar directamente dentro del seno coronario y el atrio derecho (7).

Existen otras similitudes morfológicas entre el pulmón porcino y el humano. Ambas especies tienen abundante pero variable cantidad de colágeno pleural visceral, lo que lleva a que las membranas pleurales sean más gruesas que en otras especies (22). El aporte vascular pleural visceral es de las arterias bronquiales en ambas especies, y los linfáticos pleurales viscerales son extensos (7). 


\section{Histología del pulmón porcino}

La arquitectura histológica es similar en las vías respiratorias humanas y en las porcinas. El tipo de epitelio que reviste su luz en los cerdos depende del nivel de las vías respiratorias que se analiza. Los pasajes nasales porcinos se componen de varios tipos de células epiteliales, incluidos epitelio escamoso, epitelio columnar seudoestratificado ciliado (epitelio respiratorio), epitelio sin cilios, epitelio columnar (epitelio de transición) y epitelio olfativo (31). Las líneas del epitelio respiratorio seudoestratificado de las vías respiratorias grandes y las líneas epiteliales columnares ciliadas de las vías respiratorias pequeñas de los bronquios y bronquiolos, se observan en el biomodelo porcino y en los humanos (13).

A diferencia de lo que sucede en otros animales, como los perros, en los bronquios de los cerdos se encuentra un gran número de glándulas submucosas (13). Las glándulas submucosas se asocian con la capa de músculo liso en todos los niveles bronquiales, tanto en la luz como en el cartílago. El tejido restante adyacente a las glándulas consta de cartílago y tejido conjuntivo y, probablemente, esto representa más del $80 \%$ de la masa de la pared bronquial. La capa no cartilaginosa que recubre toda la luz bronquial es rica en glándulas submucosas. La diferenciación del epitelio bronquial porcino en células ciliadas, células caliciformes y células basales ocurre a los 80 días de gestación (32); las glándulas submucosas primordiales se observan alrededor de los 92 días de gestación y continúan desarrollándose durante el tiempo de maduración (32). Se ha identificado una disminución brusca de la concentración de las glándulas submucosas en las vías respiratorias más pequeñas, más allá de la unión bronquial-bronquiolar.

El epitelio de la vía aérea porcina tiene una permeabilidad al agua relativamente alta, y el epitelio traqueal porcino absorbe espontáneamente el líquido (33). Los cultivos primarios de tejido porcino y de los epitelios de las vías respiratorias humanas muestran un transporte de electrolitos cuantitativamente similar (34).

En conclusión, el cerdo es valioso como modelo animal para condiciones patológicas de interés, así como para estudiar la viabilidad y la plausibilidad de nuevas terapias. En este sentido, el entendimiento de la anatomía del pulmón porcino y la aproximación con protocolos establecidos de investigación facilitan el aprendizaje de los grupos que están empezando programas de trasplante pulmonar.

\section{Agradecimientos}

A Gabriel Jaime Echeverri-Junca, coordinador del Laboratorio de Cirugía Experimental y Cirugía Avanzada del Centro para la Investigación en Cirugía Avanzada y Trasplantes de la Universidad Icesi.

\section{Referencias}

1. Walters EM, Wolf E, Whyte JJ, Mao J, Renner S, Nagashima H, et al. Completion of the swine genome will simplify the production of swine as a large animal biomedical model. BMC Med Genomics. 2012;5:55. https://doi.org/10.1186/1755-8794-5-55

2. Rogers CS, Abraham WM, Brogden KA, Engelhardt JF, Fisher JT, McCray PB, et al. The porcine lung as a potential model for cystic fibrosis. Am J Physiol Lung Cell Mol Physiol. 2008;295:L240-63. https://doi.org/10.1152/ajplung.90203.2008

3. Bruun CS, Jensen LK, Leifsson PS, Nielsen J, Cirera S, Jørgensen CB, et al. Functional characterization of a porcine emphysema model. Lung. 2013;191:669-75.

https://doi.org/10.1007/s00408-013-9504-2 
4. Borges JB, Costa EL, Suárez-Sipmann F, Widström C, Larsson A, Amato M, et al. Early inflammation mainly affects normally and poorly aerated lung in experimental ventilatorinduced lung injury. Crit Care Med. 2014;42:e279-87. https://doi.org/10.1097/CCM.0000000000000161

5. Kita YF, Ando A, Tanaka K, Suzuki S, Ozaki Y, Uenishi H, et al. Application of highresolution, massively parallel pyrosequencing for estimation of haplotypes and gene expression levels of swine leukocyte antigen (SLA) class I genes. Immunogenetics. 2012;64:187-99. https://doi.org/10.1007/s00251-011-0572-2

6. Snell GI, Paraskeva M, Westall GP. Donor selection and management. Semin Respir Crit Care Med. 2013;34:361-70. https://doi.org/10.1055/s-0033-1348464

7. Karimi A, Cobb JA, Staples ED, Baz MA, Beaver TM. Technical pearls for swine lung transplantation. J Surg Res. 2011;171:e107-11.https://doi.org/10.1016/j.jss.2011.05.067

8. Collins AM, Rylance J, Wootton DG, Wright AD, Wright AK, Fullerton DG, et al. Bronchoalveolar lavage (BAL) for research; obtaining adequate sample yield. J Vis Exp. 2014. https://doi.org/10.3791/4345

9. Bufalari A, De Monte V, Pecoriello R, Donati L, Ceccarelli S, Cagini L, et al. Experimental left pneumonectomy in pigs: Procedure and management. J Surg Res. 2015;198:208-16. https://doi.org/10.1016/j.jss.2015.05.045

10. Lama VN, Belperio JA, Christie JD, El-Chemaly S, Fishbein MC, Gelman AE, et al. Models of lung transplant research: A consensus statement from the National Heart, Lung, and Blood Institute workshop. JCI Insight. 2017;2:1-14. https://doi.org/10.1172/jci.insight.93121

11. Cypel M, Liu M, Rubacha M, Yeung JC, Hirayama S, Anraku M, et al. Functional repair of human donor lungs by IL-10 gene therapy. Sci Transl Med. 2009;1:1-9. https://doi.org/10.1126/scitranslmed.3000266

12. Daggett CW, Yeatman M, Lodge AJ, Chen EP, Linn SS, Gullotto C, et al. Total respiratory support from swine lungs in primate recipients. J Thorac Cardiovasc Surg. 1998;115:19-27. https://doi.org/https://doi.org/10.1016/S0022-5223(98)70438-6

13. Hartmann JF, Hutchison CF, Jewell ME. Pig bronchial mucous membrane: A model system for assessing respiratory mucus release in vitro. Exp Lung Res. 1984;6:59-70.

14. Hartert M, Senbaklavacin O, Gohrbandt B, Fischer BM, Buhl R, Vahld CF. Lung transplantation: A treatment option in end-stage lung disease. Dtsch Arztebl Int. 2014;111:107-16. https://doi.org/10.3238/arztebl.2014.0107

15. Haworth SG, Hislop AA. Adaptation of the pulmonary circulation to extra-uterine life in the pig and its relevance to the human infant. Cardiovasc Res. 1981;15:108-19.

16. Davies G, Reid L. Growth of the alveoli and pulmonary arteries in childhood. Thorax. 1970;25:669-81. https://doi.org/10.1136/thx.25.6.669

17. Florens M, Sapoval B, Filoche M. An anatomical and functional model of the human tracheobronchial tree. J Appl Physiol (1985). 2011;110:756-63. https://doi.org/10.1152/japplphysiol.00984.2010

18. Maina JN, van Gils P. Morphometric characterization of the airway and vascular systems of the lung of the domestic pig, Sus scrofa: comparison of the airway, arterial and venous systems. Comp Biochem Physiol A Mol Integr Physiol. 2001;130:781-98. https://doi.org/10.1016/S1095-6433(01)00411-1

19. Cohen BS, Sussman RG, Lippmann M. Factors affecting distribution of airflow in a human tracheobronchial cast. Respir Physiol. 1993;93:261-78.

20. Noble PB, McLaughlin RA, West AR, Becker S, Armstrong JJ, McFawn PK, et al. Distribution of airway narrowing responses across generations and at branching points, assessed in vitro by anatomical optical coherence tomography. Respir Res. 2010;11:9. https://doi.org/10.1186/1465-9921-11-9

21. Menkes HA, Macklem PT. Collateral Flow. Suppl 12. Handbook of Physiology, The Respiratory System, Mechanics of Breathing. 2011. Wiley Online Library. p. 337-53. https://doi.org/10.1002/cphy.cp030321

22. Peake JL, Pinkerton KE. Gross and subgross anatomy of lungs, pleura, connective tissue septa, distal airways, and structural units A2. In: Parent RA, Elsevier Inc. Comparative biology of the normal lung. Second edition. San Diego: Academic Press; 2015. p. 21-31. https://doi.org/10.1016/B978-0-12-404577-4.00003-5 
23. Woolcock AJ, Macklem PT. Mechanical factors influencing collateral ventilation in human, dog, and pig lungs. J Appl Physiol. 1971;30:99-115. https://doi.org/10.1152/jappl.1971.30.1.99

24. Nakakuki S. Bronchial tree, lobular division and blood vessels of the pig lung. J Vet Med Sci. 1994;56:685-9.

25. Siegel MJ, Shackelford GD, Francis RS, McAlister WH. Tracheal bronchus. Radiology. 1979;130:353-5. https://doi.org/10.1148/130.2.353

26. Dondelinger RF, Ghysels MP, Brisbois D, Donkers E, Snaps FR, Saunders J, et al. Relevant radiological anatomy of the pig as a training model in interventional radiology. Eur Radiol. 1998;8:1254-73. https://doi.org/10.1007/s003300050545

27. Schummer A, Nickel R, Sack WO. The viscera of the domestic mammals. Berlín: SpringerVerlag; 1979. p. 211- 279.

28. Amis TC, McKiernan BC. Systematic identification of endobronchial anatomy during bronchoscopy in the dog. Am J Vet Res. 1986;47:2649-57.

29. Boyden EA. Segmental anatomy of the lungs: A study of the patterns of the segmental bronchi and related pulmonary vessels. New York and London: McGraw-Hill; 1955. p. 108.

30. Judge EP, Hughes JM, Egan JJ, Maguire M, Molloy EL, O'Dea S. Anatomy and bronchoscopy of the porcine lung. A model for translational respiratory medicine. Am J Respir Cell Mol Biol. 2014;51:334-43. https://doi.org/10.1165/rcmb.2013-0453TR

31. Jones TC, Hunt RD, King NW. Veterinary pathology. Baltimore: Lippincott Williams \& Wilkins; 1997. $1392 \mathrm{p}$.

32. Baskerville A. Histological and ultrastructural observations on the development of the lung of the fetal pig. Acta Anat (Basel). 1976;95:218-33.

33. Crews A, Taylor AE, Ballard ST. Liquid transport properties of porcine tracheal epithelium. J Appl Physiol. 2001;91:797-802.

34. Liu X, Luo M, Zhang L, Ding W, Yan Z, Engelhardt JF. Bioelectric properties of chloride channels in human, pig, ferret, and mouse airway epithelia. Am J Respir Cell Mol Biol. 2007;36:313-23. https://doi.org/10.1165/rcmb.2006-02860C 